\title{
On the Complete Elliptic Integrals and Babylonian Identity IV: The Complete Elliptic Integral of first kind as sum of two Gauss hypergeometric functions
}

\author{
Edigles Guedes ${ }^{1}$ and Prof. Dr. K. Raja Rama Gandhi ${ }^{2}$ \\ Number Theorist, Brazil ${ }^{1}$ \\ and \\ Resource person in Math for Oxford University Press and Professor in Math at BITS-Vizag ${ }^{2}$ \\ edigles.guedes@gmail.com
}

\begin{abstract}
I evaluate the complete elliptic integral of first kind as the sum of two Gauss
\end{abstract} hypergeometric functions.

\section{INTRODUCTION}

In this paper, I evaluate the complete elliptic integral of first kind as the sum of two Gauss hypergeometric functions, as follows:

$$
K(k)=\frac{\pi}{2}{ }_{2} F_{1}\left(\frac{1}{2}, \frac{1}{2} ; 2 ; k^{2}\right)+\frac{\pi k^{2}}{16}{ }_{2} F_{1}\left(\frac{3}{2}, \frac{3}{2} ; 3 ; k^{2}\right) .
$$

\section{THEOREM}

Theorem 1. For $0<k<1$, then

$$
K(k)=\frac{\pi}{2}{ }_{2} F_{1}\left(\frac{1}{2}, \frac{1}{2} ; 2 ; k^{2}\right)+\frac{\pi k^{2}}{16}{ }_{2} F_{1}\left(\frac{3}{2}, \frac{3}{2} ; 3 ; k^{2}\right),
$$

where $K(k)$ is the complete elliptic integral of first kind.

Proof. In previous paper [1], Theorem 1, I proved that

$$
\frac{K(k)}{\sqrt{\pi}}=\sum_{n=0}^{\infty} \frac{\left(\frac{1}{2}\right)_{n}^{2} \Gamma\left(n+\frac{3}{2}\right)}{\left(\frac{3}{2}\right)_{n}} \frac{k^{2 n}}{n !^{2}},
$$

for $0<k<1$.

I know that

$$
\begin{gathered}
\left(\frac{1}{2}\right)_{n}^{2}=\frac{[(2 n) !]^{2}}{2^{4 n} n !^{2}} \\
\left(\frac{3}{2}\right)_{n}=\frac{(2(n+1)) !}{2^{2 n+1}(n+1) !}
\end{gathered}
$$

And

$$
\Gamma\left(n+\frac{3}{2}\right)=\sqrt{\pi} \frac{(2 n+1) !}{2^{2 n+1} n !}
$$


From (1), (2), (3) and (4), it follows that

$$
\begin{aligned}
\frac{K(k)}{\sqrt{\pi}} & =\sqrt{\pi} \sum_{n=0}^{\infty} \frac{[(2 n) !]^{2} 2^{2 n+1}(n+1) !(2 n+1) !}{2^{4 n}(2(n+1)) ! n !^{2} 2^{2 n+1} n !} \frac{k^{2 n}}{n !^{2}} \\
& =\sqrt{\pi} \sum_{n=0}^{\infty} \frac{[(2 n) !]^{2}(n+1) !(2 n+1) !}{n !^{2}(2(n+1)) ! n !} \frac{k^{2 n}}{2^{4 n} n !^{2}} \\
& =\sqrt{\pi} \sum_{n=0}^{\infty}\left(\frac{n+1}{n+2}\right) \frac{\left(\begin{array}{c}
2 n \\
n
\end{array}\right)^{2}\left(\begin{array}{c}
2 n+1 \\
n
\end{array}\right)}{\left(\begin{array}{c}
2 n+2 \\
n
\end{array}\right)}\left(\frac{k}{4}\right)^{2 n},
\end{aligned}
$$

ergo,

$$
\begin{gathered}
K(k)=\pi \sum_{n=0}^{\infty}\left(\frac{n+1}{n+2}\right) \frac{\left(\begin{array}{c}
2 n \\
n
\end{array}\right)^{2}\left(\begin{array}{c}
2 n+1 \\
n
\end{array}\right)}{\left(\begin{array}{c}
2 n+2 \\
n
\end{array}\right)}\left(\frac{k}{4}\right)^{2 n} \\
=\pi \sum_{n=0}^{\infty}\left(\frac{n}{n+2}\right) \frac{\left(\begin{array}{c}
2 n \\
n
\end{array}\right)^{2}\left(\begin{array}{c}
2 n+1 \\
n
\end{array}\right)}{\left(\begin{array}{c}
2 n+2 \\
n
\end{array}\right)}\left(\frac{k}{4}\right)^{2 n}+\pi \sum_{n=0}^{\infty}\left(\frac{1}{n+2}\right) \frac{\left(\begin{array}{c}
2 n \\
n
\end{array}\right)^{2}\left(\begin{array}{c}
2 n+1 \\
n
\end{array}\right)}{\left(\begin{array}{c}
2 n+2 \\
n
\end{array}\right)}\left(\frac{k}{4}\right)^{2 n} \\
=\frac{\pi k^{2}}{16}{ }_{2} F_{1}\left(\frac{3}{2}, \frac{3}{2} ; 3 ; k^{2}\right)+\frac{\pi}{2}{ }_{2} F_{1}\left(\frac{1}{2}, \frac{1}{2} ; 2 ; k^{2}\right) \\
=\frac{\pi}{2}{ }_{2} F_{1}\left(\frac{1}{2}, \frac{1}{2} ; 2 ; k^{2}\right)+\frac{\pi k^{2}}{16}{ }_{2} F_{1}\left(\frac{3}{2}, \frac{3}{2} ; 3 ; k^{2}\right) .
\end{gathered}
$$

Note: For the reader's delight, I construct the Table 1. 


\section{Table 1}

In this table, I have: first column: $m$; second column: $k=1 / m$; third column: $\frac{\pi}{16 m^{2}}{ }_{2} F_{1}\left(\frac{3}{2}, \frac{3}{2} ; 3 ; \frac{1}{m^{2}}\right)$; fourth column: $\frac{1}{2} \pi_{2} F_{1}\left(\frac{1}{2}, \frac{1}{2} ; 2 ; \frac{1}{m^{2}}\right)$, fifth column: $\frac{\pi}{16 m^{2}}{ }_{2} F_{1}\left(\frac{3}{2}, \frac{3}{2} ; 3 ; \frac{1}{m^{2}}\right)+$ $\frac{1}{2} \pi_{2} F_{1}\left(\frac{1}{2}, \frac{1}{2} ; 2 ; \frac{1}{m^{2}}\right)$; sixth column: $K(k)$.

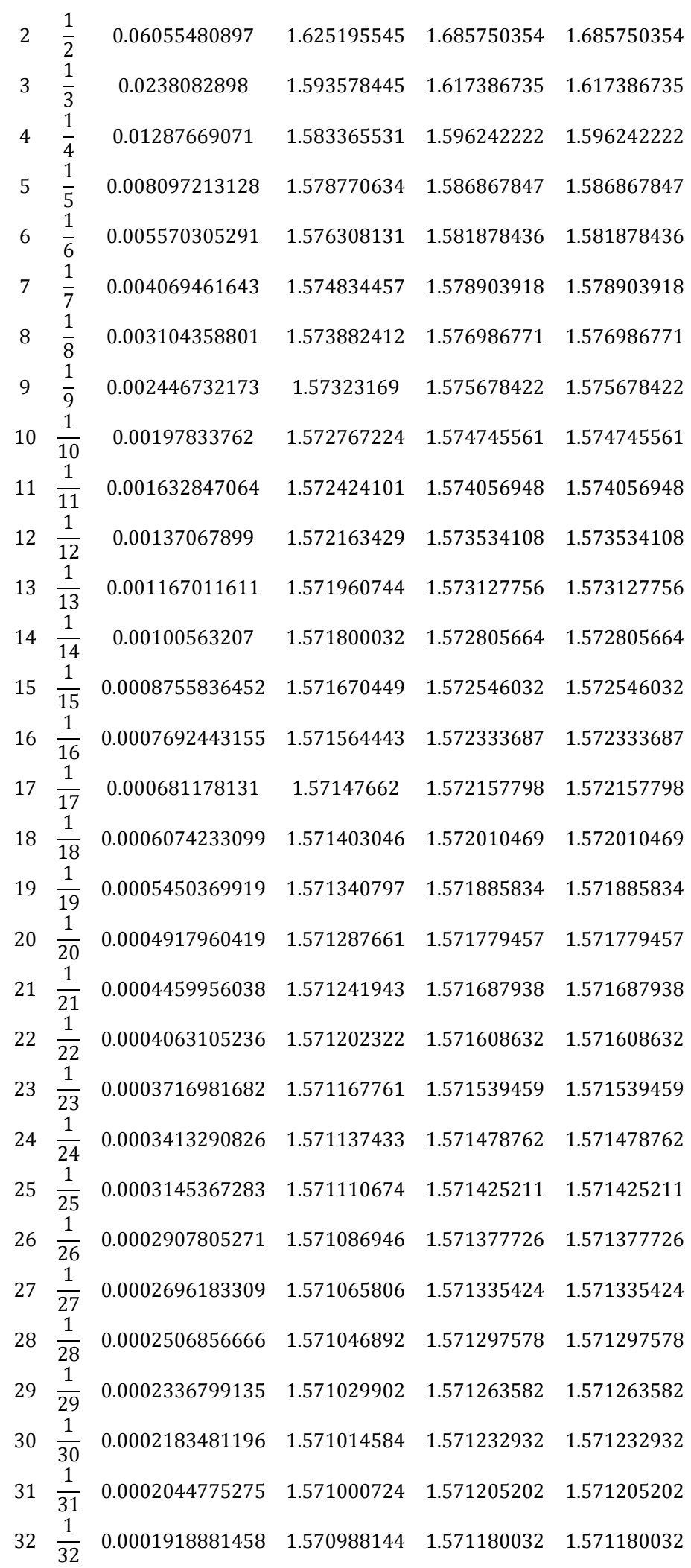




\section{REFERENCES}

[1] Guedes, Edigles, On the complete elliptic integrals and babylonian Identity 1: the $\frac{1}{\pi}$ formulaes involving gamma functions and summations, 2013. 